\title{
Evaluation of the methods for response analysis under non-stationary excitation
}

\author{
R.S. Jangid ${ }^{\mathrm{a}, *}$ and T.K. Datta ${ }^{\mathrm{b}}$ \\ ${ }^{a}$ Department of Civil Engineering, Indian Institute of \\ Technology Bombay, Powai, Mumbai - 400076, India \\ ${ }^{\mathrm{b}}$ Department of Civil Engineering, Indian Institute of \\ Technology Delhi, Hauzkhas, New Delhi-110016, \\ India
}

Received 11 December 1996

Revised 18 October 1999

Response of structures to non-stationary ground motion can be obtained either by the evolutionary spectral analysis or by the Markov approach. In certain conditions, a quasistationary analysis can also be performed. The first two methods of analysis are difficult to apply for complex situations such as problems involving soil-structure interaction, nonclassical damping and primary-secondary structure interaction. The quasi-stationary analysis, on the other hand, provides an easier solution procedure for such cases. Here-in, the effectiveness of the quasi-stationary analysis is examined with the help of the analysis of a single degree-of-freedom (SDOF) system under a set of parametric variations. For this purpose, responses of the SDOF system to uniformly modulated non-stationary random ground excitation are obtained by the three methods and they are compared. In addition, the relative computational efforts for different methods are also investigated.

Keywords: Non-stationary, earthquake, quasi-stationary, comparative performance, Markov method

\section{Introduction}

For the random vibration analyses of structures, two approaches are widely used namely, the spectral approach and the Markov approach. In the former, deterministic relation between the moments of the excitation and the response is established based on the fundamental results of mean square calculus which permit expectation and mean square limit operations to commute. In the latter, the formulation is based on the as-

\footnotetext{
${ }^{*}$ Corresponding author.
}

sumption that the response at any instant of time depends only upon the response and excitation at the previous time station. It requires a space state transition matrix that transforms one state to the immediate next state. However, the excitations need to be presented in terms of white-noise.

For the stationary random excitation, the spectral approach is generally preferred over the Markov approach, especially for multi degree-of-freedom (MDOF) system subjected to multiple support excitations. One of the major reasons for this is that the representation of many types of random excitation (like, wind and wave) in a form, which permits the Markov formulation, is difficult. However, in the case of seismic excitation, both approaches can be used and have their own advantages and disadvantages. When the earthquake is modelled as a non-stationary excitation (uniformly modulated), the Markov approach is easier to adopt for MDOF system. The spectral approach becomes difficult since the determination of the evolutionary (time dependent) frequency response function of the MDOF system is generally complicated and in certain cases cannot be analytically determined. However, a quasi-stationary spectral analysis, if applicable, becomes very easy to apply.

Lin [5] and Nigam [7] presented the general method for computing the evolutionary response using the spectral analysis for non-stationary excitation. Hammond [4] and Robert [10] obtained the response of MDOF system to non-stationary excitation using modal analysis technique and spectral approach. Perotti [8] obtained the evolutionary response of a soilstructure interaction problem with multi-point seismic excitation modelled as a non-stationary random process. Spectral analysis was used by neglecting the structural damping terms. Su and Ahmadi [13] obtained the response of a cantilever continuous beams to uniformly modulated random ground motion.

Markov approach was used by Gasparani and DebChoudhary [3] to obtain the evolutionary response of MDOF systems with and without multi-point excitation. DebChoudhary and Gazis [2] used the same 
approach for the determination of the root mean square (r.m.s.) response of MDOF system to multipoint, multi-component support excitation. Using the Markov approach, Soliman and Datta [11] obtained the evolutionary r.m.s. response of piping systems with multi-point, multi-component seismic excitation considering the flexibility of the support. The concept of quasi-stationary spectral analysis is referred by Newland [6]. Recently, Soliman and Datta [12] presented a quasi-stationary spectral analysis for obtaining the evolutionary response of piping systems with flexible support to non-stationary multiple support excitation.

The three different methods for obtaining the evolutionary response of structures subjected to nonstationary excitation have different assumptions, accuracies and computational efficiencies. The comparison of the responses obtained by the three methods is not widely reported. In particular, the validity of the quasistationary spectral analysis under different conditions has not been thoroughly investigated. In this paper, the responses of a single degree-of-freedom (SDOF) system to non-stationary earthquake excitation are obtained by the three methods and compared under a set of important parametric variations. The specific objectives of the study are to (i) investigate the effectiveness of the quasi-stationary approach for different conditions and (ii) evaluate the computational efficiency of the three methods.

\section{Model of earthquake excitation}

The earthquake excitation is considered as a uniformly modulated stationary Gaussian random process with zero-mean. The earthquake acceleration $\ddot{x}(t)$ is expressed as

$$
\ddot{x}(t)=A(t) \ddot{x}_{\mathrm{f}}(t),
$$

where $A(t)$ is the deterministic modulating function; and $\ddot{x}_{\mathrm{f}}(t)$ is the stationary random process.

The evolutionary power spectral density function (PSDF) of the earthquake excitation is given by

$$
S_{\ddot{x}}(\omega)=|A(t)|^{2} S_{\ddot{x}_{\mathrm{f}}}(\omega),
$$

where $S_{\ddot{x}_{\mathrm{f}}}(\omega)$ is the stationary PSDF of the earthquake ground motion. In the present study, the PSDF of the earthquake excitation is considered as that suggested by Clough and Penzien [1], i.e.,

$$
\begin{aligned}
S_{\ddot{x}_{\mathrm{f}}}(\omega)= & S_{0}\left(\frac{1+4 \xi_{\mathrm{g}}^{2}\left(\omega / \omega_{\mathrm{g}}\right)^{2}}{\left[1-\left(\omega / \omega_{\mathrm{g}}\right)^{2}\right]^{2}+4 \xi_{\mathrm{g}}^{2}\left(\omega / \omega_{\mathrm{g}}\right)^{2}}\right) \\
& \times\left(\frac{\left(\omega / \omega_{\mathrm{g}}\right)^{4}}{\left[1-\left(\omega / \omega_{\mathrm{f}}\right)^{2}\right]^{2}+4 \xi_{\mathrm{f}}^{2}\left(\omega / \omega_{\mathrm{f}}\right)^{2}}\right),
\end{aligned}
$$

where $S_{0}$ is the constant PSDF of input white-noise random process; $\omega_{\mathrm{g}}, \xi_{\mathrm{g}}, \omega_{\mathrm{f}}$ and $\xi_{\mathrm{f}}$ are the ground filter parameters.

Note that the Markov method requires that the excitation must be either white-noise or shot-noise whereas the PSDF of $\ddot{x}_{\mathrm{f}}(t)$ is a non-white random process. However, this obstacle can be circumvented by introducing the shaping filters in which the random process $\ddot{x}_{\mathrm{f}}(t)$ can be considered as the response of two linear filters subjected to white-noise excitation as

$$
\begin{aligned}
& \ddot{x}_{\mathrm{f}}(t)+2 \xi_{\mathrm{f}} \omega_{\mathrm{f}} \dot{x}_{\mathrm{f}}(t)+\omega_{\mathrm{f}}^{2} x_{\mathrm{f}}(t)=\ddot{x}_{\mathrm{g}}(t)+\ddot{x}_{0}(t), \\
& \ddot{x}_{\mathrm{g}}(t)+2 \xi_{\mathrm{g}} \omega_{\mathrm{g}} \dot{x}_{\mathrm{g}}(t)+\omega_{\mathrm{g}}^{2} x_{\mathrm{g}}(t)=-\ddot{x}_{0}(t),
\end{aligned}
$$

where $\ddot{x}_{0}(t)$ is the input white-noise random process with constant intensity of the PSDF as $S_{0}$. Note that the Eqs (4) and (5) provide the stationary PSDF of the response $\ddot{x}_{\mathrm{f}}(t)$ as that given by Eq. (3).

Three types of modulating functions are considered in the study namely, exponential, box-car and trapezoidal as shown in Fig. 1. The box-car type modulating function is expressed as

$$
A(t)= \begin{cases}A_{0} & \text { for } 0 \leqslant t \leqslant T_{0} \\ 0 & \text { for } t>T_{0}\end{cases}
$$

where $A_{0}$ is the scaling factor; and $T_{0}$ is the strong motion duration of the earthquake excitation.

Exponential modulating function is expressed as

$$
A(t)=A_{0}\left(\mathrm{e}^{-b_{1} t}-\mathrm{e}^{-b_{2} t}\right), \quad b_{2}>b_{1},
$$

where the values of the parameters $b_{2}$ and $b_{1}$ control the shape of the modulating function and $A_{0}$ is the scaling factor. The parameters $b_{2}$ and $b_{1}$ are defined with the help of strong motion duration $T_{0}$ and $\varepsilon$ (fraction of rise time defined later). Trifunac and Brady [14] defined $T_{0}$ as

$$
T_{0}=t_{95}-t_{5},
$$

where $t_{95}$ and $t_{5}$ are the times at which the energy content of the modulating function is $95 \%$ and $5 \%$, respec- 


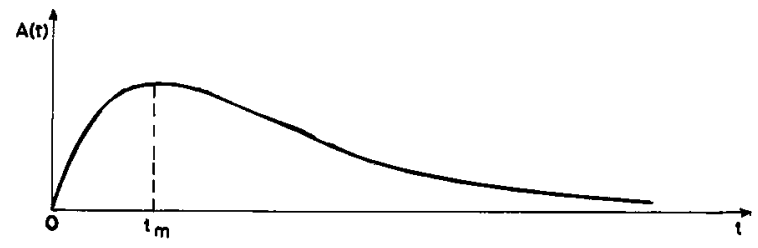

(a)

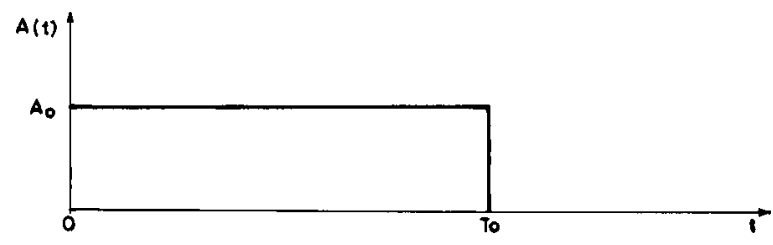

(b)

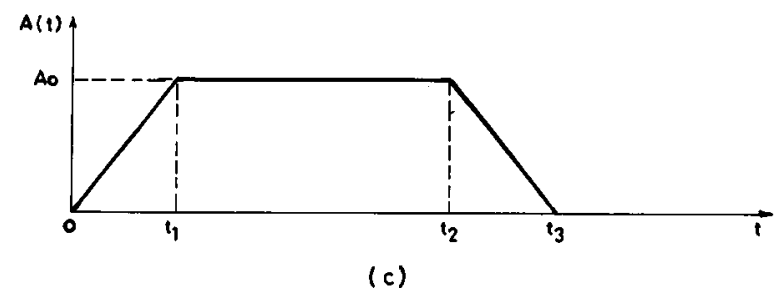

Fig. 1. Different types of modulating functions: (a) exponential, (b) box-car and (c) trapezoidal.

tively, of the total energy content. Thus, the times $t_{95}$ and $t_{5}$ are obtained by

$$
\int_{0}^{t_{95}} A^{2}(t) \mathrm{d} t=0.95 \int_{0}^{\infty} A^{2}(t) \mathrm{d} t
$$

and

$$
\int_{0}^{t_{5}} A^{2}(t) \mathrm{d} t=0.05 \int_{0}^{\infty} A^{2}(t) \mathrm{d} t .
$$

The fraction of rise time $\varepsilon$ for the exponential modulating function is defined as

$$
\varepsilon=\frac{t_{\mathrm{m}}}{t_{95}}
$$

where $t_{\mathrm{m}}$ is the time at which $A(t)$ attains the maximum value.

Using Eqs (9)-(11), a relationship can be established between the ratio $\alpha=b_{2} / b_{1}$ and $\varepsilon$. The values of parameters $b_{1}$ and $b_{2}$ can be obtained from the given values of $\varepsilon$ and $T_{0}$ as shown by Quek et al. [9].

Trapezoidal type modulating function is expressed as

$$
A(t)= \begin{cases}A_{0}\left(\frac{t}{t_{1}}\right), & 0 \leqslant t \leqslant t_{1}, \\ A_{0}, & t_{1} \leqslant t \leqslant t_{2}, \\ A_{0}\left(\frac{t-t_{3}}{t_{2}-t_{3}}\right), & t_{2} \leqslant t \leqslant t_{3}, \\ 0, & t \geqslant t_{3}\end{cases}
$$

where $A_{0}$ is the scaling factor; and $t_{1}, t_{2}$ and $t_{3}$ are the transition times of the modulating function as shown in Fig. 1(c).

The corresponding strong motion duration for the trapezoidal modulating function is taken as that given by Eq. (8). However, the fraction of rise time $\varepsilon$ is expressed as

$$
\varepsilon=\frac{t_{1}}{T_{0}}
$$

Different types of modulating functions are so scaled that the intensity $I$ defined as

$$
I=\int_{0}^{\infty}|A(t)|^{2} \mathrm{~d} t
$$

have the same value. This implies that all modulating functions have the same energy content.

In Fig. 2 different types of exponential modulating functions considered in the study are plotted for $I=$ $1 \mathrm{~s}$ and $T_{0}=5 \mathrm{~s}$. Figure shows that the exponential function becomes flatter as the parameter $\varepsilon$ increases. The corresponding parameters of the above functions are shown in Table 1 . The trapezoidal modulating function is characterised by the parameters namely $\kappa_{1}, \kappa_{2}$, $\kappa_{3}$ and $T_{0}\left(\kappa_{1}, \kappa_{2}, \kappa_{3}\right.$ are the fraction of total energy content of the modulating function between the time 0 to $t_{1}, t_{1}$ to $t_{2}$ and $t_{2}$ to $t_{3}$, respectively) for the specified

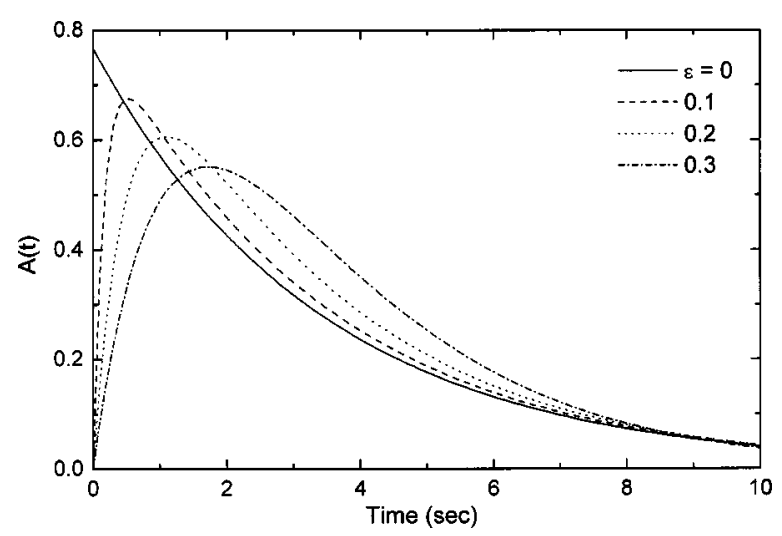

Fig. 2. Shape of different exponential modulating functions $(I=1 \mathrm{~s}$ and $T_{0}=5 \mathrm{~s}$ ). 
Table 1

Parameters of different exponential modulating functions (for $I=1 \mathrm{~s}$ and $\left.T_{0}=5 \mathrm{~s}\right)$

\begin{tabular}{lcccc}
\hline Parameters & $\varepsilon=0$ & 0.1 & 0.2 & 0.3 \\
\hline Scaling factor $A_{0}$ & 0.767 & 0.833 & 1.024 & 2.330 \\
Constant $b_{1}\left(\mathrm{~s}^{-1}\right)$ & 0.294 & 0.298 & 0.329 & 0.412 \\
Constant $b_{2}\left(\mathrm{~s}^{-1}\right)$ & $\infty$ & 5.983 & 1.989 & 0.792 \\
$\alpha=b_{2} / b_{1}$ & $\infty$ & 20.06 & 6.242 & 1.924 \\
\hline
\end{tabular}

value of $I$. Since $\kappa_{1}+\kappa_{2}+\kappa_{3}=1$ one has to specify the energy contents in two regions of the modulating function.

\section{Evolutionary response of SDOF system}

For a linear SDOF system subjected to earthquake acceleration $\ddot{x}(t)$, the relative displacement $y(t)$ of the system is governed by

$$
\ddot{y}(t)+2 \xi \omega_{0} \dot{y}(t)+\omega_{0}^{2} y(t)=-\ddot{x}(t),
$$

where $\omega_{0}$ is the natural frequency and $\xi$ is the damping coefficient of the SDOF system.

\subsection{Evolutionary spectral analysis}

For a system initially at rest and $\ddot{x}(t)$, a Gaussian random process admitting an evolutionary spectral representation of Eq. (15), it follows that $y(t)$ is also a Gaussian random process [7] given by

$$
y(t)=\int_{-\infty}^{\infty} M(t, \omega) \mathrm{e}^{\mathrm{i} \omega t} \mathrm{~d} \ddot{x}_{\mathrm{f}}(\omega),
$$

where

$$
\begin{aligned}
& M(t, \omega)=-\int_{0}^{t} h(t-\tau) A(\tau) \mathrm{e}^{-\mathrm{i} \omega(t-\tau)} \mathrm{d} \tau, \\
& h(\tau)=\frac{\mathrm{e}^{-\xi \omega_{0} \tau}}{\omega_{\mathrm{d}}} \sin \left(\omega_{\mathrm{d}} \tau\right)
\end{aligned}
$$

and

$$
\omega_{\mathrm{d}}=\omega_{0} \sqrt{1-\xi^{2}}
$$

The evolutionary PSDF of the relative displacement of the SDOF system $S_{y}(t, \omega)$ is given by

$$
S_{y}(t, \omega)=|M(t, \omega)|^{2} S_{\ddot{x}_{\mathrm{f}}}(\omega)
$$

Mean square relative displacement of the SDOF system is given by

$$
\sigma_{y}^{2}(t)=\int_{-\infty}^{\infty} S_{y}(t, \omega) \mathrm{d} \omega
$$

The $M(t, \omega)$ can be written as

$$
M(t, \omega)=M_{\mathrm{R}}(t, \omega)+\mathrm{i} M_{\mathrm{I}}(t, \omega),
$$

where $M_{\mathrm{R}}(t, \omega)$ and $M_{\mathrm{I}}(t, \omega)$ are the real and imaginary parts of $M(t, \omega)$, respectively.

For the exponential modulating function (Eq. (7)), the two parts of the $M(t, \omega)$ are expressed as

$$
\begin{aligned}
M_{\mathrm{R}}(t, \omega)= & \frac{A_{0}}{2 \omega_{\mathrm{d}}} \sum_{j=1}^{2} \sum_{i=1}^{2} \frac{(-1)^{i}}{\left(b_{i}-\xi \omega_{0}\right)^{2}+\omega_{j}^{2}} \\
& \times\left\{\mathrm { e } ^ { - \xi \omega _ { 0 } t } \left[\left(b_{i}-\xi \omega_{0}\right) \sin \omega_{j} t\right.\right. \\
& \left.\left.-\omega_{j} \cos \omega_{j} t\right]+\omega_{j} \mathrm{e}^{-b_{i} t}\right\},
\end{aligned}
$$

$$
\begin{aligned}
M_{\mathrm{I}}(t, \omega)= & \frac{A_{0}}{2 \omega_{\mathrm{d}}} \sum_{j=1}^{2} \sum_{i=1}^{2} \frac{(-1)^{i+j-1}}{\left(b_{i}-\xi \omega_{0}\right)^{2}+\omega_{j}^{2}} \\
& \times\left\{\mathrm { e } ^ { - \xi \omega _ { 0 } t } \left[\left(b_{i}-\xi \omega_{0}\right) \cos \omega_{j} t\right.\right. \\
& \left.+\omega_{j} \sin \omega_{j} t\right] \\
& \left.-\left(b_{i}-\xi \omega_{0}\right) \mathrm{e}^{-b_{i} t}\right\},
\end{aligned}
$$

where $\omega_{1}=\omega_{\mathrm{d}}+\omega$ and $\omega_{2}=\omega_{\mathrm{d}}-\omega$.

The two parts of $M(t, \omega)$ for the box-car type modulating function (Eq. (6)) are given by 
$M_{\mathrm{R}}(t, \omega)=\left\{\begin{aligned} \frac{A_{0}}{2 \omega_{\mathrm{d}}} \sum_{j=1}^{2} \frac{-1}{\xi^{2} \omega_{0}^{2}+\omega_{j}^{2}} \\ \quad \times\left\{\mathrm{e}^{-\xi \omega_{0} t}\left[-\xi \omega_{0} \sin \omega_{j} t\right.\right. \\ \left.\left.\quad-\omega_{j} \cos \omega_{j} t\right]+\omega_{j}\right\}, \\ 0 \leqslant t \leqslant T_{0}, \\ \frac{A_{0} \mathrm{e}^{-\xi \omega_{0} t} \sum_{j=1}^{2} \frac{-1}{\xi^{2} \omega_{0}^{2}+\omega_{j}^{2}}}{2 \omega_{\mathrm{d}}} \\ \quad \times\left\{-\xi \omega_{0} \sin \omega_{j} t-\omega_{j} \cos \omega_{j} t\right. \\ -\mathrm{e}^{-\xi \omega_{0} T_{0}}\left[-\xi \omega_{0} \sin \omega_{j}\left(t-T_{0}\right)\right. \\ \left.\left.-\omega_{j} \cos \omega_{j}\left(t-T_{0}\right)\right]\right\}, \\ t>T_{0},\end{aligned}\right.$

$$
M_{\mathbf{I}}(t, \omega)=\left\{\begin{array}{l}
\frac{A_{0}}{2 \omega_{\mathrm{d}}} \sum_{j=1}^{2} \frac{(-1)^{j}}{\xi^{2} \omega_{0}^{2}+\omega_{j}^{2}} \\
\quad \times\left\{\mathrm { e } ^ { - \xi \omega _ { 0 } t } \left[-\xi \omega_{0} \cos \omega_{j} t\right.\right. \\
\left.\left.\quad+\omega_{j} \sin \omega_{j} t\right]+\xi \omega_{0}\right\}, \\
0 \leqslant t \leqslant T_{0}, \\
\frac{A_{0} \mathrm{e}^{-\xi \omega_{0} t} \sum_{j=1}^{2} \frac{(-1)^{j}}{\xi^{2} \omega_{0}^{2}+\omega_{j}^{2}}}{2 \omega_{\mathrm{d}}} \\
\quad \times\left\{-\xi \omega_{0} \cos \omega_{j} t+\omega_{j} \sin \omega_{j} t\right. \\
\quad-\mathrm{e}^{-\xi \omega_{0} T_{0}}\left[-\xi \omega_{0} \cos \omega_{j}\left(t-T_{0}\right)\right. \\
\left.\left.\quad+\omega_{j} \sin \omega_{j}\left(t-T_{0}\right)\right]\right\}, \\
t>T_{0} .
\end{array}\right.
$$

The corresponding expression of $M_{\mathrm{R}}(t, \omega)$ and $M_{\mathrm{I}}(t, \omega)$ for the trapezoidal modulating function are given in the Appendix.

\subsection{Markov method}

An alternative approach for determination of the response statistics is to form expectations by direct manipulation on the equations of motion of the system. This is most conveniently carried out using the state variable formulation. Equation (15) along with Eqs (1), (4), (5) can be re-written as a system of first order stochastic differential equations as

$$
\frac{\mathrm{d}}{\mathrm{d} t}\{z\}=[H]\{z\}+\{F\},
$$

where

$$
\{z\}=\left\{y(t), \dot{y}(t), x_{\mathrm{f}}(t), \dot{x}_{\mathrm{f}}(t), x_{\mathrm{g}}(t), \dot{x}_{\mathrm{g}}(t)\right\}^{\mathrm{T}},
$$

$$
[H]=\left[\begin{array}{cccc}
0 & 1 & 0 & \\
-\omega_{0}^{2} & -2 \xi \omega_{0} & \omega_{\mathrm{f}}^{2} A(t) & \\
0 & 0 & 0 & \\
0 & 0 & -\omega_{\mathrm{f}}^{2} & \\
0 & 0 & 0 & \\
0 & 0 & 0 & \\
0 & 0 & 0 \\
2 \xi_{\mathrm{f}} \omega_{\mathrm{f}} A(t) & \omega_{\mathrm{g}}^{2} A(t) & 2 \xi_{\mathrm{g}} \omega_{\mathrm{g}} A(t) \\
1 & 0 & 0 \\
-2 \xi_{\mathrm{f}} \omega_{\mathrm{f}} & -\omega_{\mathrm{g}}^{2} & -2 \xi_{\mathrm{g}} \omega_{\mathrm{g}} \\
0 & 0 & 1 \\
0 & -\omega_{\mathrm{g}}^{2} & -2 \xi_{\mathrm{g}} \omega_{\mathrm{g}}
\end{array}\right]
$$

and

$$
\{F\}=\left\{0,0,0,0,0,-\ddot{x}_{0}(t)\right\}^{\mathrm{T}} .
$$

The augmented response vector $\{z\}$ is a Markov process and the corresponding covariance matrix $[\mathrm{V}]$ satisfies the following differential equation [7]:

$$
\frac{\mathrm{d}}{\mathrm{d} t}[V]=[H][V]+[V][H]^{\mathrm{T}}+[P],
$$

where $[H]^{\mathrm{T}}$ is the transpose of the matrix $[H]$ and the elements of the covariance matrix $[V]$ are given by

$$
V_{i j}=E\left[z_{i} z_{j}\right], \quad i, j=1,2, \ldots, 6 .
$$

The elements of the matrix $[P], P_{i j}=0$ except $P_{66}=2 \pi S_{0}$.

The non-stationary response of the system (i.e., $[V]$ matrix) is obtained by solving the moment equation (31). The mean square displacement of the SDOF system $\sigma_{y}^{2}(t)$ will be the element $V_{11}$ of the matrix [ $\left.V\right]$ at any instant of time. The augmented system matrix $[H]$ is time dependent through the introduction of the modulating function $A(t)$. Therefore, Eq. (31) is to be solved numerically based on step-by-step method. The fourth order Runge-Kutta method is employed for the present study. The initial covariance matrix for the system $\left[V_{0}\right]$ considered for obtaining the response of the system is as follows

$$
\left[V_{0}\right]=\left[\begin{array}{cc}
0 & 0 \\
0 & V_{\mathrm{f}}^{S}
\end{array}\right],
$$

where $V_{\mathrm{f}}^{S}$ is the matrix of size $(4 \times 4)$ containing the stationary response of ground filters; and 0 is the null matrix of respective size due to initial at rest condition of the SDOF system. Note that $V_{\mathrm{f}}^{S}$ ensures that the 
earthquake excitation reaches to its stationary condition before it is multiplied by the modulating function (refer Eq. (1)).

\subsection{Quasi-stationary analysis}

In the quasi-stationary analysis, the convolution of impulse response function with the modulating function is not performed to derive the evolutionary frequency response represented by Eq. (17). Instead, the evolutionary PSDF of the response is obtained as

$$
S_{y}(t, \omega)=A(t)^{2}|H(\omega)|^{2} S_{\ddot{x}_{\mathrm{f}}}(\omega)
$$

where $H(\omega)=\left(-\omega^{2}+\mathrm{i} 2 \xi \omega_{0} \omega+\omega_{0}^{2}\right)^{-1}$ is the frequency response function of linear SDOF system which is the Fourier transform of the impulse response function $h(\tau)$.

The mean square displacement of the system by quasi-stationary analysis is obtained from Eqs (21) along with (34). Further, by comparing Eqs (20) and (34), it can be shown that in the quasi-stationary analysis the following assumption is made

$$
|M(t, \omega)|^{2}=A(t)^{2}|H(\omega)|^{2} .
$$

This assumption leads to substantial reduction in the computational efforts especially for the MDOF system. Further, it also allows in many cases the determination of an approximate evolutionary response of structural system to non-stationary excitation where exact solution is difficult to obtain [12]. However, it remains to be examined to what extent the simplified solution as given by Eq. (35) is valid under different conditions.

\section{Numerical study}

Responses of the SDOF system to non-stationary earthquake excitation are obtained by three different methods and are compared under different important parametric variations in order to investigate (i) the effectiveness of the quasi-stationary analysis, (ii) computational efficiencies of the methods, and (iii) the effects of important parameters on the response. The important parameters which are considered include: strong motion duration of earthquake excitation $\left(T_{0}\right)$, the ratio of rise time of modulating function to the earthquake duration $(\varepsilon)$ for the exponential and the trapezoidal functions and the damping ratio of the SDOF system $(\xi)$. In the present study, the period of the SDOF system (i.e., $\left.2 \pi / \omega_{0}\right)$ is taken as $1 \mathrm{~s}$ and kept constant throughout. The modulating functions are scaled in such a way that their energy contents expressed by Eq. (14) are equal to $1 \mathrm{~s}$. The time step for integration of equations in Markov method has been taken as $0.01 \mathrm{~s}$ and the frequency step taken for obtaining the mean square response by Eq. (21) is considered to be $0.1 \mathrm{rad} / \mathrm{s}$. The time step of integration is kept small (about 1/100 of the fundamental time period of the SDOF system) in order to get sufficient accuracy of the response analysis. This accuracy has been checked by performing the analysis by varying the time step between 0.001 to $0.1 \mathrm{~s}$. Similarly, the frequency step is decided after checking the results of the numerical integration of Eq. (21). The fraction of energy content of the trapezoidal function in the region between $t_{2}$ to $t_{3}$ (i.e., $\kappa_{3}$ ) is taken as 0.1 . The filter parameters for the PSDF of earthquake excitation are taken as $\omega_{\mathrm{g}}=15 \mathrm{rad} / \mathrm{s}, \omega_{\mathrm{f}}=0.1 \omega_{\mathrm{g}}, \xi_{\mathrm{g}}=\xi_{\mathrm{f}}=0.6$ and $S_{0}=0.01 \mathrm{~m}^{2} /\left(\mathrm{s}^{3} \mathrm{rad}\right)$. These parameters correspond to the earthquake ground motion for a firm soil [1]. In addition, the performance of the quasi-stationary method for two triangular type modulating functions on the response of the system is also investigated.

Figure 3 compares the evolutionary r.m.s. displacement obtained by the three methods for different types of modulating functions. The parameters considered are $T_{0}=5 \mathrm{~s}$ and $\xi=5 \%$. It is seen from the figure that the spectral analysis and the Markov method give exactly the same responses for all cases. Note that the Markov method obtains the evolutionary value of the r.m.s. response by the numerical integration of Eq. (31) which relates the covariance of response to the covariance of excitations at each time station. The results of the integration depend upon the time step and the initial conditions assumed. The spectral analysis obtains the evolutionary PSDF of the response through closed form expressions given by Eq. (20). The expressions include the initial conditions through the impulse response function $h(\tau)$ (appearing in Eqs (16)(18)). Same initial conditions have been considered for both the methods. The quasi-stationary method provides different r.m.s. response for all modulating functions. However, this method provides conservative estimate of the peak value of the response.

In Table 2 relative computational efficiency of various methods normalised with respect to spectral method is shown. The Markov and quasi-stationary methods take considerably less time than that of the spectral method. Also, it was observed that the CPU time taken by spectral method is significantly de- 

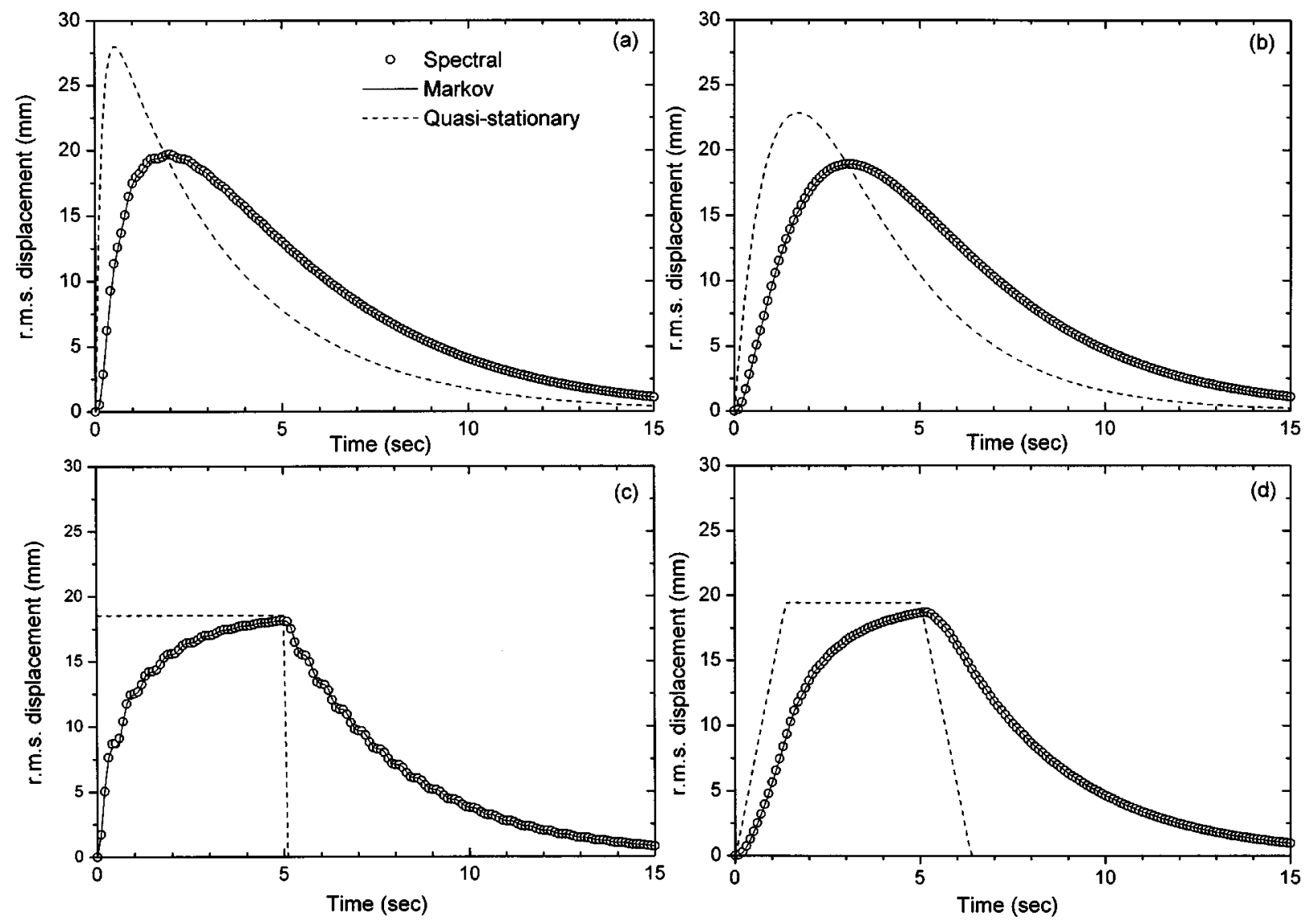

Fig. 3. Time variation of r.m.s displacement of SDOF system by different methods for $T_{0}=5 \mathrm{~s}$ and $\xi=5 \%$ : (a) exponential with $\varepsilon=0.1$, (b) exponential with $\varepsilon=0.3$, (c) box-car and (d) trapezoidal with $\kappa_{1}=0.1$.

Table 2

Relative computational time efficiency of various methods

\begin{tabular}{lccc}
\hline Method & \multicolumn{3}{c}{ CPU time/ $t_{s}$} \\
\cline { 2 - 4 } & Exponential & Box-car & Trapezoidal \\
\hline Spectral & 1 & 1 & 1 \\
Markov & 0.208 & 0.666 & 0.107 \\
Quasi-stationary & 0.016 & 0.037 & 0.008 \\
\hline
\end{tabular}

$t_{s}=$ CPU time of the spectral method.

pendent on the shape of the modulating function. It was significantly higher for the trapezoidal function in comparison to the exponential and box-car functions. This is due to fact that the expression for $M(t, \omega)$ for trapezoidal function is relatively more complicated (refer Appendix and Eqs (23)-(26)). On the other hand, the CPU time consumed by the Markov and quasi-stationary methods is not much influenced by the shape of modulating function. Thus, one can prefer the Markov method which is computationally more efficient for obtaining the exact non-stationary response of the system in comparison to spectral method for any type of modulating functions (provided the excitation is expressed in terms of white-noise). Further, the quasi-stationary method can be applied at the cost of the accuracy of the response with considerably less computational efforts.

Since spectral and Markov methods provide the same response of the system, as a result, in the further study the effectiveness of quasi-stationary method is investigated by comparing the corresponding response by the Markov method.

Figure 4 shows the variation of the peak r.m.s. displacement with the strong motion duration of the excitation $T_{0}$. The error in the peak r.m.s. displacement obtained by the quasi-stationary method is quite significant for short duration earthquake. However, as the duration of earthquake increases, the error decreases. For the duration of earthquake greater than 10 s (i.e., 10 times the period of SDOF system), the quasi-stationary method provides nearly the same response as those 

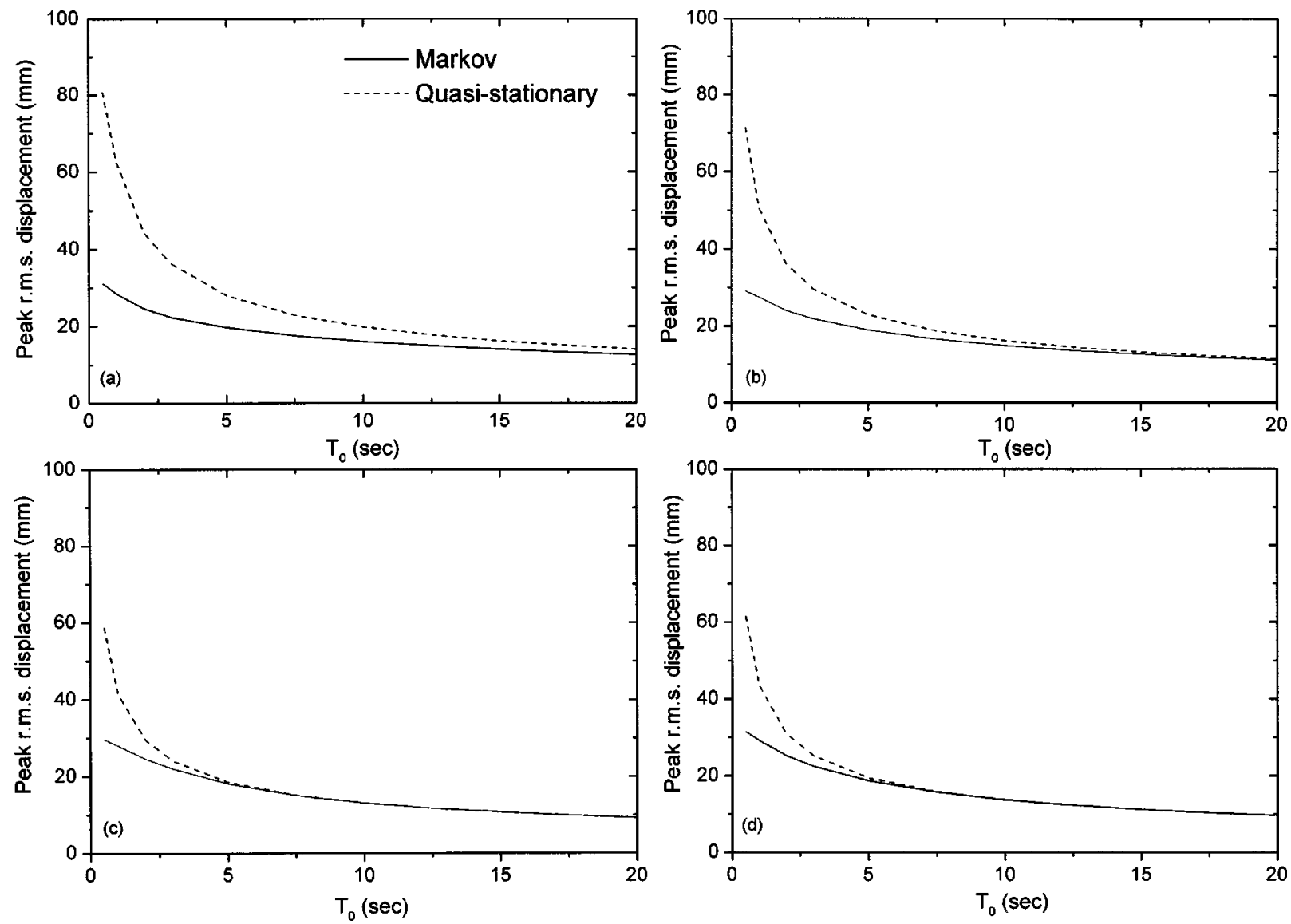

Fig. 4. Effects of strong motion duration of earthquake ground motion on the peak r.m.s displacement of the SDOF system for $\xi=5 \%$ : (a) exponential with $\varepsilon=0.1$, (b) exponential with $\varepsilon=0.3$, (c) box-car and (d) trapezoidal with $\kappa_{1}=0.1$.

given by the Markov method for the exponential type modulating functions. For the box-car and trapezoidal type modulating functions, this requirement on the duration of earthquake is about $5 \mathrm{~s}$. Thus, the quasistationary method provides better values of peak r.m.s. response for longer duration of modulating functions. Further, it is also observed from the Fig. 4 that the peak r.m.s. displacement of the system by the Markov method is almost same for all modulating functions. This implies that for the same duration, the shape of modulating functions does not significantly influence the response of the system.

In Figure 5, the variation of the peak r.m.s. displacement is plotted against the damping ratio of the SDOF system. The strong motion duration of the modulating function is taken as $5 \mathrm{~s}$. Figure 5 indicates that the difference between the peak r.m.s. response obtained by the quasi-stationary and the Markov method decreases with the increase in the damping of the system. Thus, the quasi-stationary method provides better values of peak response for higher damping ratios of the system.

Figure 6 shows the variation of the peak r.m.s. displacement of the system against the parameter $\varepsilon$ of the exponential and trapezoidal function for $T_{0}=5 \mathrm{~s}$ and $\xi=5 \%$. For the exponential modulating, the peak r.m.s. response by the Markov method is appears to be the same for all values of $\varepsilon$ (although the shape of the modulating function considerably changes with $\varepsilon$ as shown in Fig. 2). Thus, the peak response of the system for exponential modulating functions is not influenced by the fraction of rise time provided their energy content and duration are the same. Further, for the quasi-stationary method, the peak r.m.s. response varies almost linearly with $\varepsilon$ and it tends to converge to the exact value as $\varepsilon$ increases. The effectiveness of quasi-stationary method for the trapezoidal function is not much influenced by the parameter $\varepsilon$ as shown in Fig. 6(b). 

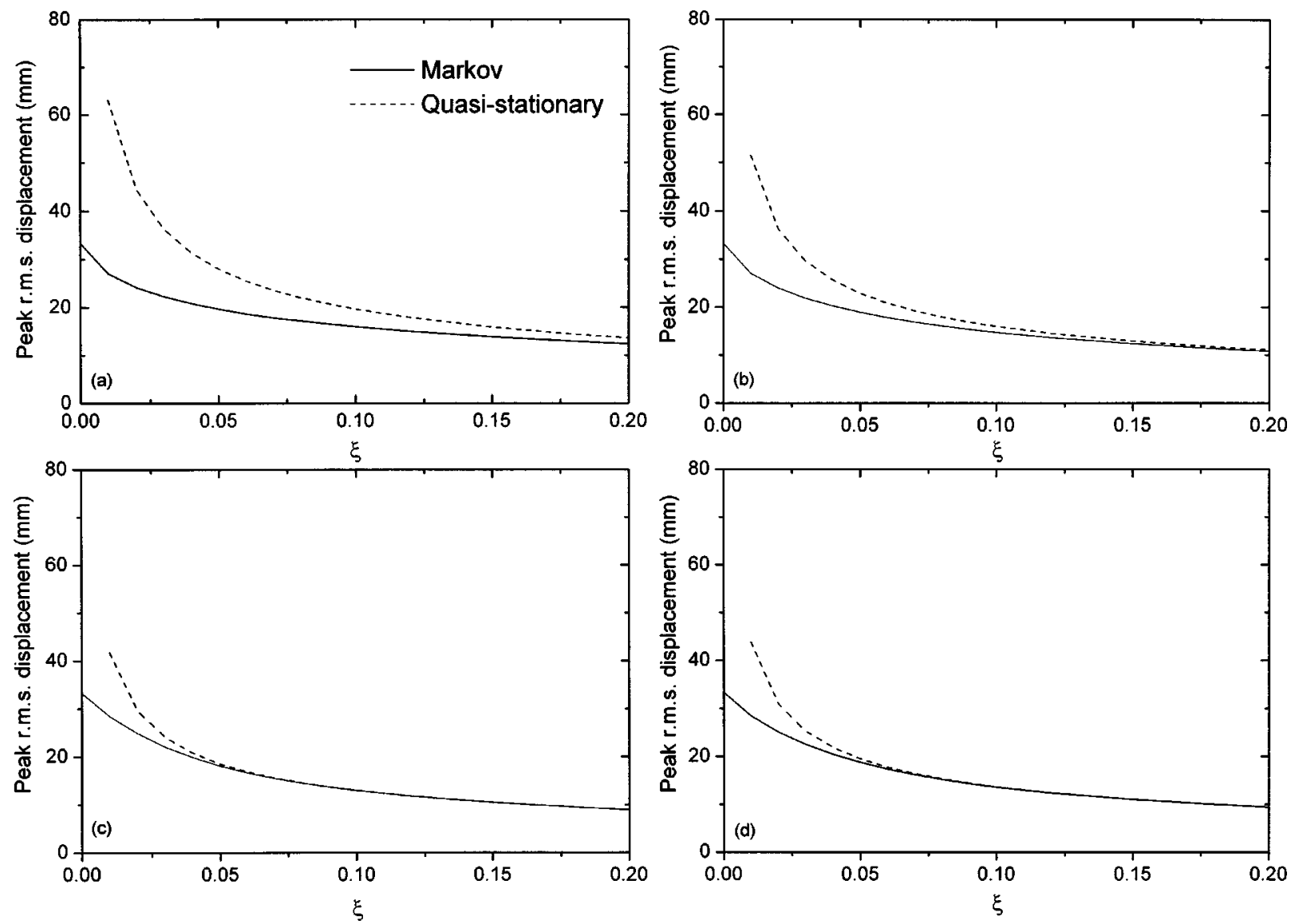

Fig. 5. Effect of damping ratio of the SDOF system on the peak r.m.s displacement for $T_{0}=5 \mathrm{~s}$ : (a) exponential with $\varepsilon=0.1$, (b) exponential with $\varepsilon=0.3$, (c) box-car and (d) trapezoidal with $\kappa_{1}=0.1$.
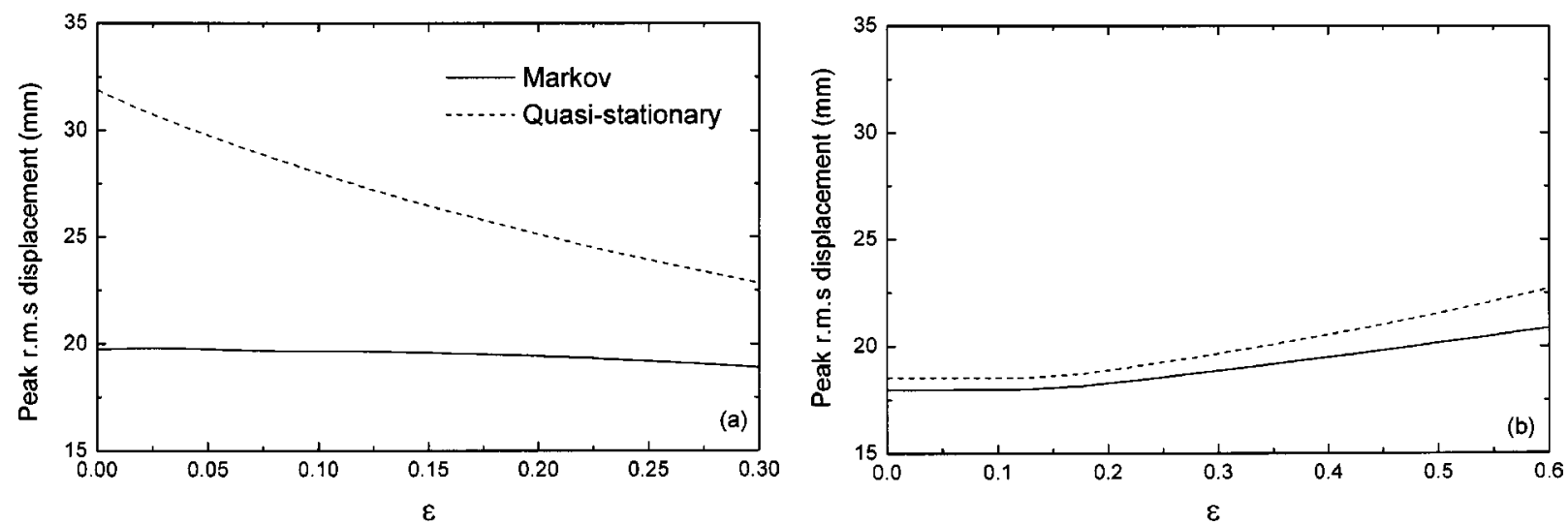

Fig. 6. Effect of rise time of modulating function on the peak r.m.s displacement of the SDOF system for $T_{0}=5 \mathrm{~s}$ and $\xi=5 \%$ : (a) exponential and (b) trapezoidal.

It will be interesting to compare the response of quasi-stationary and Markov methods for the two triangular modulating functions referred as type I and II as shown in Fig. 7 (type I is typically modelled for blast loading). These functions can be completely defined by the time duration $t_{0}$ for a specified value of energy 


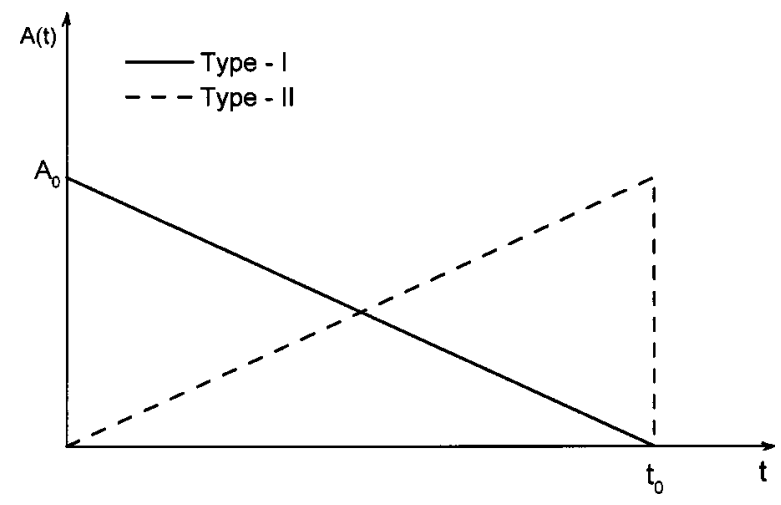

Fig. 7. Triangular modulating functions of type I and II.

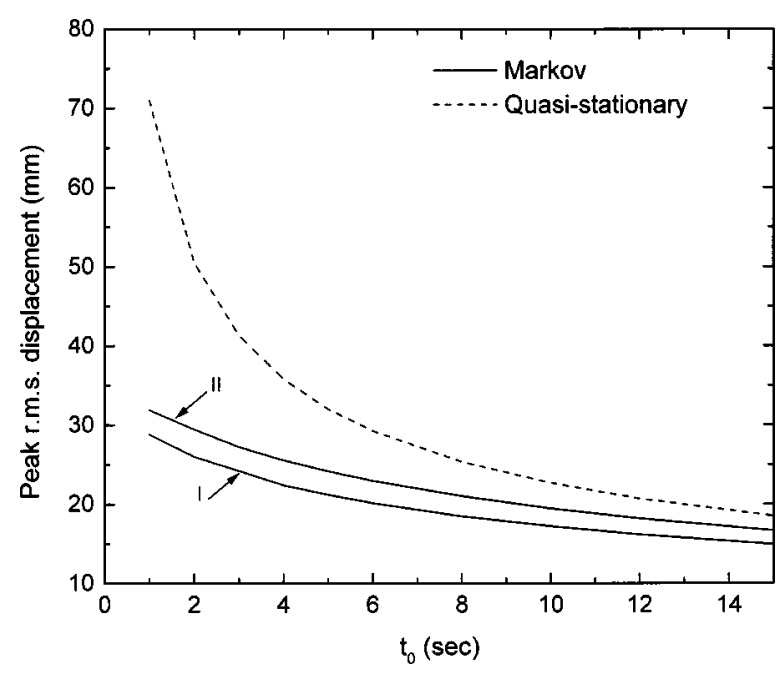

Fig. 8. Comparative performance of quasi-stationary method for triangular modulating functions.

content $I$. Note that the quasi-stationary method will provide the same result for the two modulating functions (since it is only dependent on the peak value of the modulating function). In Fig. 8 the peak r.m.s. response of the SDOF system to two triangular modulating functions is shown against the time duration $t_{0}$. As expected the difference in the response between two methods decreases with the increase of $t_{0}$. However, the error in the response by quasi-stationary method is relatively more for the triangular type I than that of II for all values of $t_{0}$. This implies that the quasistationary method provides better results for slowly increasing modulating functions than that of decreasing functions.

Note that for very short duration impulse, the quasistationary analysis over-estimates the response by about 50 to $100 \%$. For other cases also, it was observed that the quasi-stationary response over-predicts the response by about 50 to $200 \%$ (refer Figs 4 and 5) for very short duration of the strong motion excitation. In such cases, the quasi-stationary analysis should be avoided as far as possible although it may provide safe solution. Practicality of the design with such over safe quantities is to be weighted against the advantages of the simplicity of the method. Perhaps a more detailed study needs to be carried out in order to arrive at suitable scaling factors to reduce the response obtained by the quasi-stationary analysis.

\section{Conclusions}

The response of a single degree-of-freedom system to uniformly modulated non-stationary ground motion is obtained by three different methods namely, (i) evolutionary spectral analysis, (ii) Markov method and (iii) quasi-stationary method. The responses obtained by the three methods are compared for a number of important parametric variations in order to investigate their computational efficiencies and the effectiveness of the quasi-stationary analysis. From the trends of the results of the present study, the following conclusions may be drawn:

1. The evolutionary spectral method and the Markov method provide the same response for different types of modulating functions. However, for the system in the present study the Markov method is found to be computationally more efficient.

2. The quasi-stationary method is found to be computationally very efficient and provides a conservative estimate of the peak values of the response.

3. For slowly varying modulating function, the peak responses obtained by the quasi-stationary method are fairly accurate. The quasi-stationary method may be preferred for slowly increasing functions over the slowly decreasing one.

4. The quasi-stationary method provides better values of peak response for longer duration of modulating function and higher damping ratio of the system.

5. For the exponential type modulating function the peak response of the system is not significantly influenced by its shape provided their energy content and duration are the same. 


\section{Appendix}

The corresponding expression for the trapezoidal function are expressed as

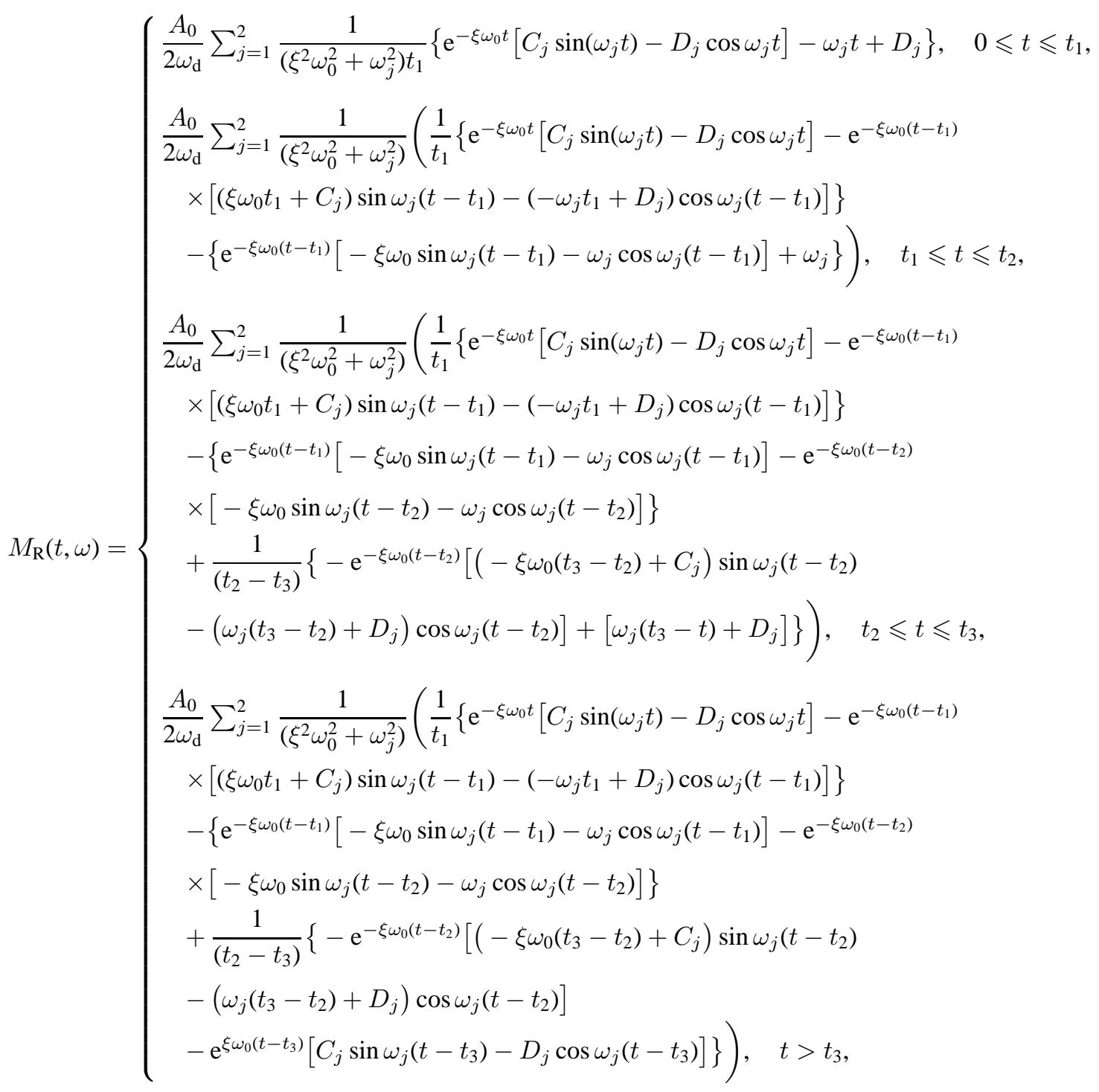

where

$$
C_{j}=\frac{\omega_{j}^{2}-\xi^{2} \omega_{0}^{2}}{\omega_{j}^{2}+\xi^{2} \omega_{0}^{2}}
$$

and

$$
D_{j}=\frac{2 \omega_{j} \xi \omega_{0}}{\omega_{j}^{2}+\xi^{2} \omega_{0}^{2}}
$$

and 


$$
\begin{aligned}
& \int \frac{A_{0}}{2 \omega_{\mathrm{d}}} \sum_{j=1}^{2} \frac{(-1)^{j+1}}{\left(\xi^{2} \omega_{0}^{2}+\omega_{j}^{2}\right) t_{1}}\left\{\mathrm{e}^{-\xi \omega_{0} t}\left[C_{j} \cos \left(\omega_{j} t\right)+D_{j} \sin \left(\omega_{j} t\right)\right]-\xi \omega_{0} t-C_{j}\right\}, \quad 0 \leqslant t \leqslant t_{1}, \\
& \frac{A_{0}}{2 \omega_{\mathrm{d}}} \sum_{j=1}^{2} \frac{(-1)^{j+1}}{\left(\xi^{2} \omega_{0}^{2}+\omega_{j}^{2}\right)}\left(\frac { 1 } { t _ { 1 } } \left\{\mathrm{e}^{-\xi \omega_{0} t}\left[C_{j} \cos \left(\omega_{j} t\right)+D_{j} \sin \left(\omega_{j} t\right)\right]-\mathrm{e}^{-\xi \omega_{0}\left(t-t_{1}\right)}\right.\right. \\
& \left.\times\left[\left(\xi \omega_{0} t_{1}+C_{j}\right) \cos \omega_{j}\left(t-t_{1}\right)+\left(-\omega_{j} t_{1}+D_{j}\right) \sin \omega_{j}\left(t-t_{1}\right)\right]\right\} \\
& \left.-\left\{\mathrm{e}^{-\xi \omega_{0}\left(t-t_{1}\right)}\left[-\xi \omega_{0} \cos \omega_{j}\left(t-t_{1}\right)+\omega_{j} \sin \omega_{j}\left(t-t_{1}\right)\right]+\xi \omega_{0}\right\}\right), \quad t_{1} \leqslant t \leqslant t_{2}, \\
& \frac{A_{0}}{2 \omega_{\mathrm{d}}} \sum_{j=1}^{2} \frac{(-1)^{j+1}}{\left(\xi^{2} \omega_{0}^{2}+\omega_{j}^{2}\right) t_{1}}\left(\frac { 1 } { t _ { 1 } } \left\{\mathrm{e}^{-\xi \omega_{0} t}\left[C_{j} \cos \left(\omega_{j} t\right)+D_{j} \sin \left(\omega_{j} t\right)\right]-\mathrm{e}^{-\xi \omega_{0}\left(t-t_{1}\right)}\right.\right. \\
& \left.\times\left[\left(\xi \omega_{0} t_{1}+C_{j}\right) \cos \omega_{j}\left(t-t_{1}\right)+\left(-\omega_{j} t_{1}+D_{j}\right) \sin \omega_{j}\left(t-t_{1}\right)\right]\right\} \\
& -\left\{\mathrm{e}^{-\xi \omega_{0}\left(t-t_{1}\right)}\left[-\xi \omega_{0} \cos \omega_{j}\left(t-t_{1}\right)+\omega_{j} \sin \omega_{j}\left(t-t_{1}\right)\right]-\mathrm{e}^{-\xi \omega_{0}\left(t-t_{2}\right)}\right. \\
& M_{\mathrm{I}}(t, \omega)=\left\{\begin{array}{l}
\left.\times\left[-\xi \omega_{0} \cos \omega_{j}\left(t-t_{2}\right)+\omega_{j} \sin \omega_{j}\left(t-t_{2}\right)\right]\right\} \\
+\frac{1}{\left(t_{2}-t_{3}\right)}\left\{-\mathrm{e}^{-\xi \omega_{0}\left(t-t_{2}\right)}\left[\left(-\xi \omega_{0}\left(t_{3}-t_{2}\right)+C_{j}\right) \cos \omega_{j}\left(t-t_{2}\right)\right.\right.
\end{array}\right. \\
& \left.\left.\left.+\left(\omega_{j}\left(t_{3}-t_{2}\right)+D_{j}\right) \sin \omega_{j}\left(t-t_{2}\right)\right]+\xi \omega_{0}\left(t_{3}-t\right)-C_{j}\right\}\right), \quad t_{2} \leqslant t \leqslant t_{3}, \\
& \frac{A_{0}}{2 \omega_{\mathrm{d}}} \sum_{j=1}^{2} \frac{(-1)^{j+1}}{\left(\xi^{2} \omega_{0}^{2}+\omega_{j}^{2}\right) t_{1}}\left(\frac { 1 } { t _ { 1 } } \left\{\mathrm{e}^{-\xi \omega_{0} t}\left[C_{j} \cos \left(\omega_{j} t\right)+D_{j} \sin \left(\omega_{j} t\right)\right]-\mathrm{e}^{-\xi \omega_{0}\left(t-t_{1}\right)}\right.\right. \\
& \left.\times\left[\left(\xi \omega_{0} t_{1}+C_{j}\right) \cos \omega_{j}\left(t-t_{1}\right)+\left(-\omega_{j} t_{1}+D_{j}\right) \sin \omega_{j}\left(t-t_{1}\right)\right]\right\} \\
& -\left\{\mathrm{e}^{-\xi \omega_{0}\left(t-t_{1}\right)}\left[-\xi \omega_{0} \cos \omega_{j}\left(t-t_{1}\right)+\omega_{j} \sin \omega_{j}\left(t-t_{1}\right)\right]-\mathrm{e}^{-\xi \omega_{0}\left(t-t_{2}\right)}\right. \\
& \left.\times\left[-\xi \omega_{0} \cos \omega_{j}\left(t-t_{2}\right)+\omega_{j} \sin \omega_{j}\left(t-t_{2}\right)\right]\right\} \\
& +\left\{\mathrm{e}^{-\xi \omega_{0}\left(t-t_{2}\right)}\left[\left(-\xi \omega_{0}\left(t_{3}-t_{2}\right)+C_{j}\right) \cos \omega_{j}\left(t-t_{2}\right)+\left(\omega_{j}\left(t_{3}-t_{2}\right)+D_{j}\right) \sin \omega_{j}\left(t-t_{2}\right)\right]\right. \\
& \left.\left.-\mathrm{e}^{-\xi \omega_{0}\left(t-t_{3}\right)}\left[C_{j} \cos \omega_{j}\left(t-t_{3}\right)+D_{j} \sin \omega_{j}\left(t-t_{3}\right)\right]\right\}\right), \quad t>t_{3} .
\end{aligned}
$$

\section{References}

[1] R.W. Clough and J. Penzien, Dynamics of Structures, McGrawHill, New York, 1993.

[2] A. DebChoudhary and G.D. Gazis, Response of MDOF system to multiple support seismic excitation, J. of Engineering Mechanics ASCE 114 (1988), 583-603.

[3] D.A. Gasparani and A. DebChoudhary, Dynamic response to non-stationary non-white excitation, J. of Engineering $\mathrm{Me}$ chanics ASCE 106 (1980), 1233-1246.

[4] J.K. Hammond, On the response of single and multiple degree of freedom systems to non-stationary excitations, J. of Sound and Vibration 7 (1968), 393-416.

[5] Y.K. Lin, Probabilistic Theory of Structural Dynamics, McGraw-Hill, New York, 1967.

[6] D.E. Newland, An Introduction to Random Vibrations and Spectral Analysis, Longman, London, 1984.
[7] N.C. Nigam, Introduction to Random Vibrations, MIT Press, Cambridge, 1983.

[8] F. Perotti, Structural response to non-stationary multiplesupport excitation, Earthquake Engineering and Structural Dynamics 19 (1990), 513-523.

[9] S.E. Quek, Y.P. Teo and T. Balendra, Non-stationary structural response with evolutionary spectra using seismological input model, Earthquake Engineering and Structural Dynamics 19 (1990), 275-288.

[10] J.B. Robert, The covariance response of linear system to nonstationary random excitations, J. of Sound and Vibration 14 (1971), 393-416.

[11] H.O. Soliman and T.K. Datta, The seismic response of a piping system to non-stationary random ground motion, J. of Sound and Vibration 180 (1995), 459-473.

[12] H.O. Soliman and T.K. Datta, Stochastic response of piping systems with flexible supports, J. of Pressure Vessel Tech. ASME 118 (1996), 109-114. 
[13] L. Su and G. Ahmadi, Earthquake response of linear continuous structures by the method of evolutionary spectra, Engineering Structures 10 (1988), 47-56.
[14] M.D. Trifunac and A.G. Brady, A study on the duration of strong earthquake ground motion, Bulletin of Seismological Society of America 65 (1975), 581-626. 

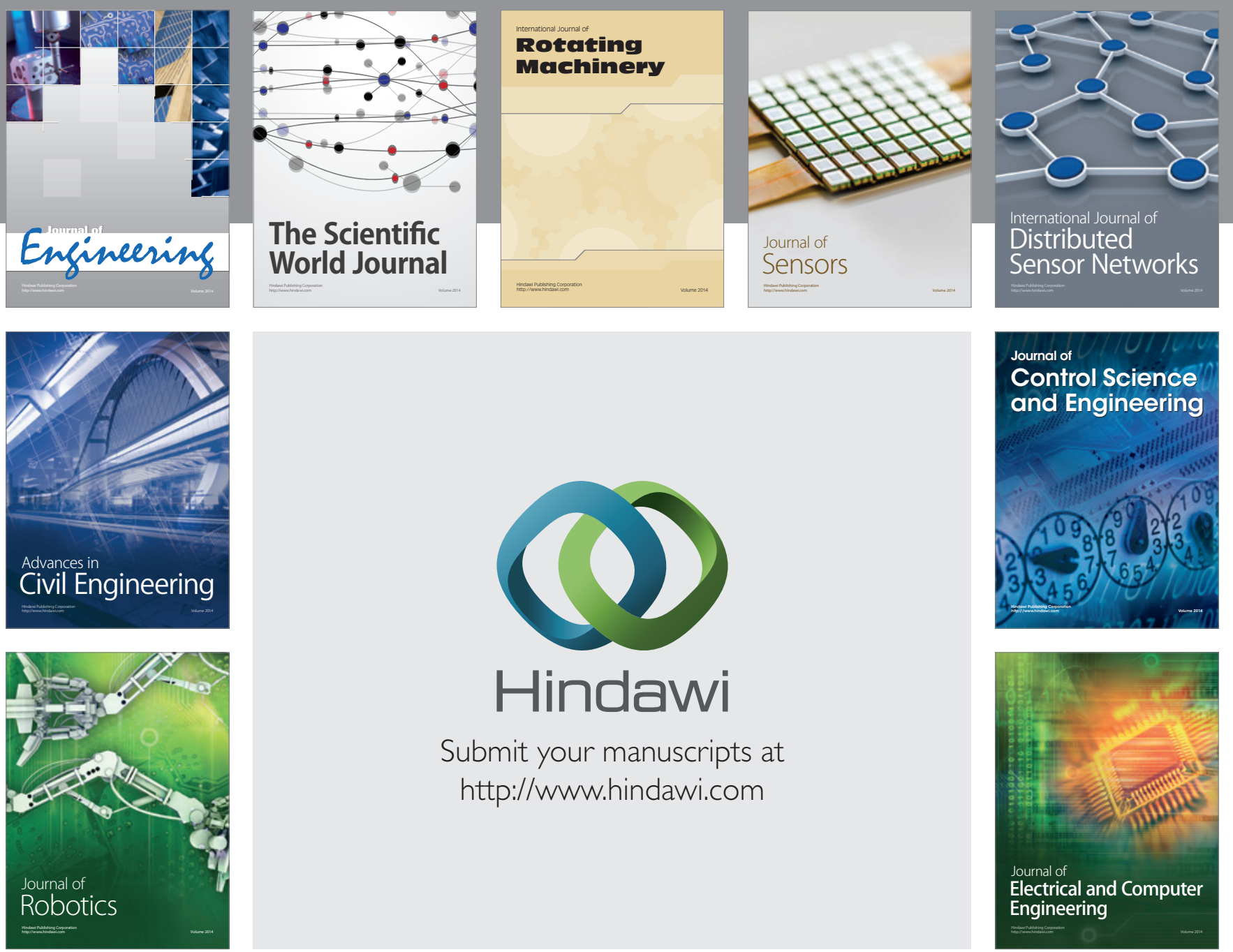

Submit your manuscripts at

http://www.hindawi.com
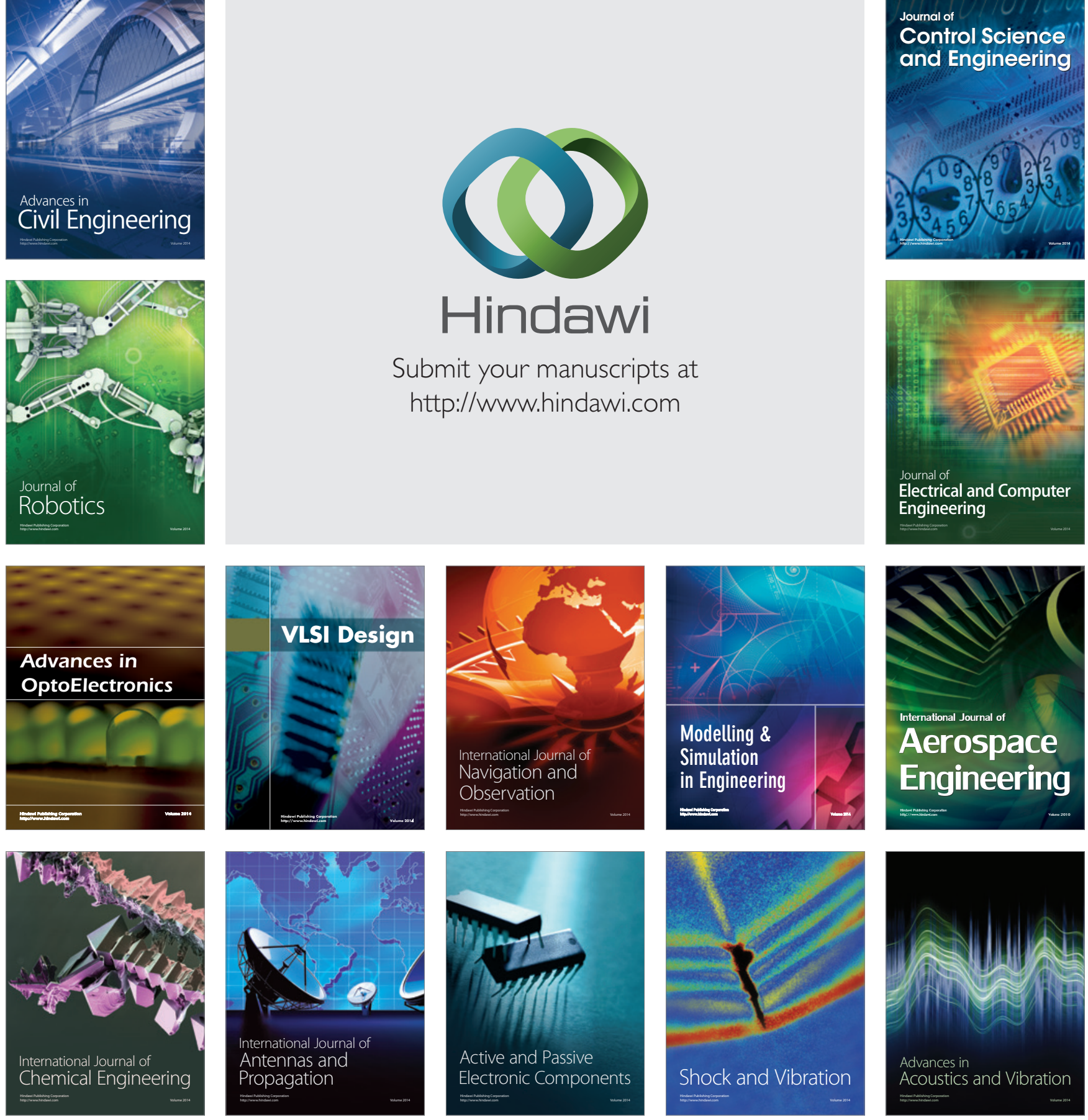